\title{
Perfiles de aprendizaje y trabajo colaborativo en entornos de simulación en 3D
}

\section{Learning profiles and collaborative work in 3D-simulation environment}

\author{
Byron Ernesto Vaca-Barahona \\ Escuela Superior Politécnica de Chimborazo (Ecuador) \\ Jose Cela-Ranilla \\ Universitat Rovira i Virgili (España) \\ Eliana Esther Gallardo-Echenique \\ Universidad Continental (Perú)
}

\section{Resumen}

Los entornos de simulación $3 \mathrm{D}$ con mediación pedagógica favorecen la comunicación e interacción de los estudiantes inmersos en la actividad formativa en el contexto de la secuencia pedagógica planteada, promoviendo así de manera natural el aprendizaje de los estudiantes, generándose dos tipos de comunicación: interacción entre participante y objeto $3 \mathrm{D}$, e interacción del tipo participante con otro participante que en la experiencia es la que se desarrolla más espontáneamente y en mayor cantidad. Se evidencia que el comportamiento y la actuación de los perfiles Strong-Willed y Bridge son distintos. El perfil Strong-Willed se caracteriza por organizar, asumir y distribuir responsabilidades, tomar decisiones, colaborar, liderar el trabajo del grupo, ser persistente y constante. Sin embargo, el perfil Bridge se caracteriza por ser comunicativo, expresivo y motivador del grupo; se apoya en el conocimiento de los miembros del grupo para aprender y desarrollar sus actividades y carece de continuidad durante el desarrollo del trabajo grupal.

Palabras clave: aprendizaje colaborativo; aprendizaje interactivo; simulación por computadora; comunicación mediada por computadora; realidad virtual.

\begin{abstract}
$3 \mathrm{D}$ simulation environments with pedagogical mediation encourage communication and interaction of students of the training activity in the corresponding sequence by promoting their learning. Two types of communication are generated: interaction between participant and object $3 \mathrm{D}$, and interaction between participants, which in practice has demonstrated to be more prolific and spontaneous. There is evidence that the behavior and performance of the Strong-Willed and Bridge profiles are different. The Strong-Willed profile is a learner
\end{abstract}


who is organized, assumes and distributes responsibilities, makes decisions, collaborates, leads the group work, and is persistent and consistent. The Bridge profile is a learner who is communicative, expressive and motivates the group members; relies on the knowledge of the group members to learn and develop his/her activities, and lacks continuity during group work.

Keywords: cooperative learning; interactive learning; computer simulation; computer mediated communication; virtual reality.

En los últimos años, se ha producido una revolución en las tecnologías de la información y comunicación (TIC) que ha ampliado las posibilidades de comunicación que anteriormente ofrecían las tecnologías tradicionales; y de manera particular, han impactado los procesos de enseñanza y de aprendizaje, las estrategias de enseñanza, los roles que desempeñan los docentes y los estudiantes, etc. (Gallardo-Echenique, 2012; Maldonado, 2007). Nos encontramos ante un mundo donde el impacto de las TIC están presentes en la mayoría de las actividades de nuestra vida diaria y contribuyen con los cambios que se producen en el trabajo, la familia y la educación, entre otros (Gallardo-Echenique, 2012). Su introducción en el ámbito educativo viene enmarcada por una situación de cambios en los docentes y estudiantes, en los entornos o escenarios de aprendizaje, en sus modelos y concepciones. Nuevas y emergentes tecnologías "ofrecen la posibilidad de transformar las aulas en entornos de aprendizaje más atractivos, colaborativos y productivos que pueden personalizar según las necesidades específicas, los intereses y las preferencias de aprendizaje del estudiante" (Gallardo-Echenique, Marqués y Bullen, 2014, p. 192).

En este marco, ¿qué pueden aportar las TIC a los procesos de trabajo colaborativo de los estudiantes y cómo estos entornos promueven un aprendizaje acorde al perfil de aprendizaje de los mismos? Para responder a dicha cuestión es necesario primero establecer la diferencia que existe entre los términos aprendizaje y trabajo colaborativo de forma general, para luego conceptualizar sus estilos de aprendizaje.

\section{EL APRENDIZAJE Y TRABAJO COLABORATIVO}

"El aprendizaje colaborativo es el uso instruccional de pequeños grupos que permite a los estudiantes trabajar juntos para maximizar su propio aprendizaje y el de los demás" (Johnson, Johnson y Johnson, 1994). El ambiente de aprendizaje colaborativo es el conjunto de métodos de enseñanza y entrenamiento que se apoya en tecnologías y estrategias para desarrollar habilidades relacionadas con el aprendizaje y con el desarrollo personal y social, donde cada participante del grupo es responsable de su aprendizaje, pero también contribuye al aprendizaje de los miembros del grupo (Lucero, 2006). Martínez y Prendes (2004) definen el aprendizaje colaborativo como una herramienta potente de innovación educativa, 
que se produce cuando se utilizan métodos de enseñanza basados en el trabajo colaborativo.

El trabajo colaborativo tiene como finalidad que el estudiante construya por sí mismo nuevo conocimiento basado en conocimientos previos, mediante apoyo e interacción con otros miembros del grupo (Johnson y Johnson, 2004). Diferentes teorías del aprendizaje le asignan un alto valor formativo al trabajo colaborativo, dado que su aplicación se basa en la interacción social (Lucero, 2006; Maldonado, 2007).

Según estas definiciones, se puede establecer que un aprendizaje colaborativo es completamente diferente a realizar un trabajo en grupo, porque un trabajo colaborativo implica contribuir con tareas que en conjunto llevan a la obtención de una meta común (Johnson y Johnson, 2004; Medina y Hernández, 2011). Su premisa básica es la construcción del consenso en donde se comparte la autoridad, y entre todos, se acepta la responsabilidad de las acciones del grupo; siendo sus habilidades sociales indispensables para desarrollar una interacción de calidad (Maldonado, 2007).

\section{El aprendizaje y trabajo colaborativo virtual}

La implementación del aprendizaje y trabajo colaborativo virtual ha sido posible gracias al desarrollo vertiginoso de las TIC (Guiza, 2011). Actualmente, las herramientas Web 2.o, además de implementar el trabajo colaborativo, proporcionan plataformas que permiten enlazar infinidad de tecnologías, actividades y recursos educativos, promoviendo el aprendizaje activo y potenciando la comunicación e interacción entre alumnos y docentes (Guiza, 2011; Vega et al., 2011).

$\mathrm{El}$ aprendizaje colaborativo virtual hace énfasis en la comunicación orientada a tareas por medio de las tecnologías de la información y comunicación. La integración de herramientas para colaboración en la web, proporciona apoyo a la comunicación y desarrollo de enlaces a otros recursos que permiten nuevas dimensiones para el aprendizaje colaborativo (Guiza, 2011). Se trata de herramientas que permiten superar las barreras que en determinados momentos provoca la falta de contacto visual y auditivo (Domínguez y Alonso, 2012).

\section{APRENDIZAJE EN ENTORNOS VIRTUALES QUE INTEGRAN SIMULACIONES 3D}

Los modelos educativos contemporáneos hacen énfasis en la importancia de la participación activa de los estudiantes como el centro del proceso de enseñanza y aprendizaje. Se hace necesario entonces hacer una revisión de las teorías de aprendizaje donde, por un lado, están los que piensan como Cela-Ranilla (2009, p. 32) que "el conocimiento es una creación mental independiente de las interacciones 
con el contexto y con los demás miembros de la sociedad" y, por otro están aquellos que creen que "el proceso de aprendizaje se produce principalmente a través de la interacción con el contexto" (p. 38).

Sin embargo, ninguna de las teorías por sí sola responde a las diferentes maneras de aprender de las personas; por lo que preferimos acercarnos al constructivismo piagetiano, así como a la teoría sociocultural del aprendizaje de Vygotsky, donde la persona aprende a través de una construcción personal como resultado de la interacción con otros y con el medio o contexto en el que se halla (Maldonado, 2007; Vaca, 2012).

Existe una gran variedad de nomenclaturas para hablar de los entornos virtuales de enseñanza y aprendizaje (EVEA); se suelen usar términos como Entornos Virtuales de Aprendizaje, Sistemas de Gestión de Aprendizaje, Plataformas de Teleformación, entre otros; sin embargo para esta investigación trabajaremos con LMS (Learning Management System), que es el término más extendido para la formación en red (Vega et al., 2011).

Se trata de facilitar el desarrollo de procesos formativos coherentes con un modelo de aprendizaje caracterizado por la construcción conjunta del aprendizaje a través de entornos tecnológicos avanzados como LMS, SLOODLE y MUVE. Moodle (utilizado en esta investigación) basa su desarrollo en la llamada "pedagogía constructivista social” (Hernández, Pennesi, Sobrino y Vázquez, 2013). Este valor añadido, observado por los educadores (De Oliveira, Gallardo-Echenique, Bettencourt y Gisbert, 2012), está relacionado con las potencialidades de interacción características del entorno de simulación, que redefine de manera poderosa las relaciones y las formas de interacción entre el profesor y los estudiantes.

\section{SISTEMA AVANZADO DE APRENDIZAJE LET ME LEARN@}

Let Me Learn $囚$ (LML) es un sistema avanzado de aprendizaje que enseña a las personas a descubrir la forma en la que cada uno aprende y a usarla con intención. Es un sistema desarrollado desde hace más de veinte años, cuando un grupo de investigadores académicos y profesionales de la educación trataron de comprender el origen y el potencial del aprendizaje intencional (Dawkins, Kottkamp y Johnston, 2010). Este sistema ha sido probado en miles de personas alrededor del mundo y ha sido creado por los Drs. Christine Johnston y Gary Dainton de la Universidad de Rowan en New Jersey.

\section{Patrones de Aprendizaje}

Paralelamente a la idea de construcción conjunta del aprendizaje, va ligada la idea de crear un espacio donde los estudiantes puedan aprender de manera activa $\mathrm{y}$, sobre todo, de la forma que mejor se adapta a la manera natural de aprender de cada uno. El Modelo de Aprendizaje Interactivo (Interactive Learning Model - ILM) 
de Johnston (1996) es coherente con la concepción de construcción activa y conjunta del aprendizaje, con una participación de los componentes cognitivo, conativo y afectivo (Dawkins, Kottkamp y Johnston, 2010):

- Proceso Cognitivo: Busca usar la inteligencia, el pensamiento, el análisis, el razonamiento, el entendimiento de palabras, imágenes, comunicación, animaciones para aprender. En el cerebro se da una convergencia de los aprendizajes del pasado con lo desconocido de lo nuevo, se invocan a experiencias y aprendizajes anteriores para crear el mayor número de asociaciones con lo nuevo que se está aprendiendo.

- Proceso Conativo: Mientras ocurre el proceso cognitivo, el cerebro de manera paralela está buscando la forma para que ocurra el aprendizaje; es decir, el cerebro simultáneamente también se prepara para actuar. El factor conativo establece la velocidad con la que se llevan a cabo las tareas de aprendizaje, y pueden ser observadas como procesos secuenciales, confluentes, técnicos o precisos.

- Proceso Afectivo: Está relacionado con las sensaciones de éxito del individuo en procesos de aprendizaje en el pasado. Cuando alguien que aprende alcanza éxito en la realización de las tareas, el cerebro libera endorfinas que dan la sensación de triunfo y logro; lo que aumenta la autoestima y la confianza en el individuo, motivando el aprendizaje en próximas experiencias. Este proceso crea seguridad y confianza en la capacidad de aprender de las personas.

Figura 1. Procesos mentales que operan en los patrones de aprendizaje

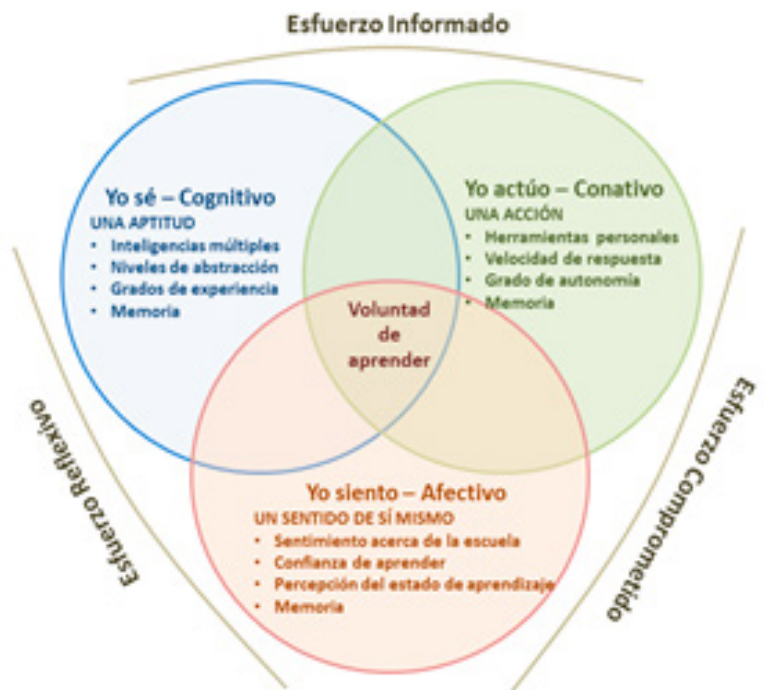

Adaptado de "Intentional teaching: The let me learn® classroom in action”, por Dawkins et al., 2010. 
Las combinaciones cognitivas, conativas y afectivas de las personas (figura 1) crean en cada persona un perfil único de aprendizaje a partir de cuatro patrones definidos:

\section{Patrón Secuencial}

Quien aprende requiere de un conjunto de direcciones claras y ordenadas que guíen el desarrollo del trabajo o actividad que se tenga que realizar. Este patrón "es la parte que hace conexiones en su aprendizaje" (Villamizar, 2006, p. 237).

\section{Patrón Precisión}

Quien aprende busca y procesa información detallada de manera muy metódica y precisa; apunta y resalta los aspectos importantes; pregunta y busca respuestas precisas y concretas; y, expresa el deseo de aprender y conocer cosas con mucha certeza.

\section{Patrón Razonamiento Técnico}

Quien aprende refleja el deseo de trabajar con el fin de entender las cosas; se puede decir, que refleja la parte práctica de lo que se aprende, se relacionan los saberes con su utilidad para resolver problemas.

\section{Patrón de Confluencia}

Quien aprende refleja la necesidad de poner las ideas en práctica, busca probar su imaginación en la resolución de un determinado problema. Se inicia incluso antes de tener definidas todas las instrucciones para la realización de la tarea. Una característica importante es su capacidad de abstracción llevándolo al borde de sus experiencias y más allá.

\section{METODOLOGÍA}

\section{Aproximación metodológica}

La investigación es de carácter cualitativo dado que se construye el conocimiento a partir de los sucesivos hallazgos que se van encontrando durante el proceso. Se caracteriza porque intenta comprender el contexto particular en el que los participantes actúan y la influencia que ese contexto tiene sobre sus acciones (Della Porta y Keating, 2008; Maxwell, 2009). En un estudio cualitativo, el investigador no 
sólo está interesado en los eventos físicos y el comportamiento que tiene lugar, sino también en la forma en que los participantes le dan sentido al estudio y cómo sus conocimientos influyen en su comportamiento (Maxwell, 2009).

El diseño se concreta en el análisis de casos, constituido por un grupo concreto de estudiantes inmersos en una actividad formativa en un entorno de simulación 3D. Para su análisis, se emplearon métodos de carácter exploratorio y descriptivo con el fin de comprender y analizar en profundidad aquellos aspectos relacionados con la interacción con propósito colaborativo generado en el entorno de simulación en $3 \mathrm{D}$.

Esta investigación se ha llevado a cabo en el marco de un proyecto de investigación financiado en el Plan Nacional de I+D+I del Ministerio de Ciencia e Innovación de España: SIMUL@: "Evaluación de Entornos Tecnológicos de simulación para el aprendizaje de competencias transversales en la Universidad" (Ref.: EDU200801479).

\section{Objeto de estudio}

Lo constituyen los "Entornos virtuales de aprendizaje que integran simulaciones $3 \mathrm{D}$ " con la finalidad de analizar la colaboración de los estudiantes y explorar la manera en que estos entornos promueven un aprendizaje acorde al perfil de aprendizaje de los estudiantes.

\section{Instrumento de Evaluación: Inventario de Conexiones de Aprendizaje}

Para caracterizar a una persona en relación a sus preferencias y predisposición para aprender, usamos el Inventario de Conexiones de Aprendizaje (Learning Combination Inventory - LCI) que es una herramienta que determina en qué grado la persona utiliza cada patrón, para esto el estudiante responde a 28 preguntas de selección múltiple y a tres preguntas de respuesta corta que sirven para validar el cuestionario (Johnston y Dainton, 1996).

Posteriormente, se cuantifican los resultados de los cuestionarios para caracterizar los patrones de aprendizaje de cada persona en dependencia de los valores obtenidos. Considerando que: Evita un patrón si la puntuación está entre 7 a 17; Usa si necesita con puntuación de 18 a 24; y, Usa primero con puntuación de 25 a 35 .

La combinación de los distintos patrones configuran perfiles específicos de aprendizaje que Johnston y Dainton (1996) han caracterizado en los siguientes términos: 


\section{Perfil Dynamic (Dinámico)}

Si un estudiante utiliza uno o dos de sus patrones en el primer nivel de uso y cualquier otra combinación de los patrones, es un aprendiz dinámico. Se caracteriza por:

- No se toma las cosas de manera particular ni asume responsabilidades solo.

- Tampoco es un buen mediador de la comunicación entre los demás.

- Asume responsabilidades específicas si se le asigna.

- Su trabajo es el reflejo de los demás miembros del grupo.

\section{Perfil Bridge (Puente)}

Si un estudiante no evita ninguno de los patrones y tampoco usa ningún patrón en el primer nivel. Se caracteriza por:

- Aprende de escuchar a los demás e interactuar con ellos.

- Se siente cómodo con todos los patrones.

- Se siente como un "aprendiz de todo y maestro de nada".

- Encontrará que puede combinarse, arrimar el hombro y ayudar a que las cosas sucedan como miembro activo del grupo.

- Coloca en la balanza las cosas antes de actuar.

- Motiva a los demás desde el centro para animar a otros en lugar de hacerse cargo de la situación.

\section{Perfil Strong-Willed (Voluntad Fuerte)}

Si un estudiante usa tres o más patrones en el primer nivel. Se caracteriza por:

- El estudiante es su propio equipo.

- Prefiere trabajar solo para poder controlar el plan, las ideas, las conversaciones, las decisiones, el proceso y los resultados.

- Para otros puede ser difícil de seguir su ejemplo.

\section{Etapas de desarrollo de la investigación}

El proyecto se desarrolló en tres etapas orientadas a dar respuesta a las preguntas y objetivos planteados en el proyecto. En la primera etapa, el esfuerzo se centra en 
la recolección de datos de las diferentes fuentes de información. En la segunda, se analizan los datos, se clasifican y se agrupan para observar y describir los aspectos comunicativos generados por la actividad de los estudiantes. Y, en la tercera, se buscan evidencias de los datos relacionados con los participantes de la investigación para determinar si estos aspectos comunicativos dan información sobre una forma natural de aprender de los estudiantes.

\section{Participantes}

En esta investigación participaron 72 estudiantes (20 hombres y 52 mujeres) del Grado de Educación Infantil, Educación Primaria y Educación Física, además de un Máster en Dirección de Marketing. De estos 72 estudiantes, se seleccionaron aquellos que tienen su perfil de aprendizaje Strong-Willed y Bridge claramente definido, utilizando el criterio de inclusión basado en las puntuaciones derivadas de la administración del LCI.

Para que un estudiante sea considerado como un perfil: (a) Bridge, era necesario que al responder el cuestionario LCI, los valores correspondientes a sus patrones estén en el rango de 18 a 24. De la población 4 estudiantes cumplen con este requisito; y (b) Strong-Willed, los valores correspondientes para al menos 3 de sus patrones debían estar en el rango de 25 a 35. Para este caso de estudio, se seleccionaron estudiantes que tuvieron puntuaciones altos en tres de sus patrones, pero que para cada caso se evite un patrón distinto, por lo que se obtienen 4 estudiantes. Con las restricciones presentadas, los participantes seleccionados fueron 8 de los cuales 6 son mujeres y 2 son hombres.

\section{Recolección y análisis de datos}

Los datos son recogidos sin modificarlos, para que sean examinados e interpretados, para luego escribir las conclusiones resultado del análisis reflexivo de lo observado.

Se inició el proceso perfilando el contexto de trabajo para responder a las siguientes interrogantes:

\section{¿Qué vamos a observar?}

- La interacción con propósito de colaboración de los estudiantes en entornos de simulación mediados pedagógicamente.

- Diferenciar la interacción colaborativa de los estudiantes en correspondencia a sus perfiles de aprendizaje. 


\section{¿Cómo se va a realizar?}

- Inicialmente, se describe el entorno virtual de aprendizaje que integra simulaciones 3D desplegado en el proyecto Simul@ para identificar las herramientas y los objetos $3 \mathrm{D}$ que se integraron para promover la interacción de los participantes con fines colaborativos.

- Luego se verificaron los diferentes registros de información y datos relacionados con el proyecto Simul@, tales como la documentación del proyecto y las bases de datos de las plataformas tecnológicas.

- Se obtienen, clasifican y agrupan los datos para su análisis.

- Se concluye la presentación y explicación de lo observado.

\section{¿Dónde se va a llevar a cabo la observación?}

- En la plataforma tecnológica desplegada para el desarrollo del proyecto Simul@ que integra Moodle con OpenSim a través de Sloodle.

\section{¿En qué tiempo se llevará a cabo la observación?}

- Para la observación, se verificaron los registros de actuación de los estudiantes durante todo el tiempo planificado para el desarrollo de la secuencia pedagógica, que fue de 3 semanas.

Además, se han diseñado instrumentos que facilitan la cuantificación de la interacción entre participantes con propósito colaborativo, y un instrumento orientado al registro y agrupamiento de las evidencias textuales de las interacciones colaborativas.

Para el análisis de datos, se realizaron las siguientes acciones para poder describir en profundidad aquellos aspectos relacionados con los objetivos del proyecto.

- Se analizaron las fuentes documentales del proyecto Simul@ en busca de información relevante de los participantes: biodatos, patrones de aprendizaje y conformación de grupos de trabajo.

- Se estudia la secuencia pedagógica planteada para el desarrollo de los proyectos de los diferentes grupos.

- Se estudia la documentación técnica relacionada con la plataforma tecnológica integrada en el proyecto Simul@, para comprender, describir su funcionalidad e identificar herramientas Moodle y Objetos 3D integrados. 
- Se analiza el contenido de las interacciones colaborativas generadas por los participantes en el contexto de la secuencia pedagógica para su cuantificación y clasificación, diferenciándola por el perfil de aprendizaje.

- Se presentan y describen los resultados de la observación.

El análisis del contenido de las interacciones se realizó a través del software libre Edet (versión 2.o.x) para el análisis cualitativo asistido por computadora.

\section{Propuesta Formativa}

Se realizó bajo una estrategia de trabajo por proyectos que los estudiantes debían desarrollar en grupo. El responsable del diseño de la actividad formativa fue el docente tutor del grupo de estudiantes participantes (tabla 1).

Tabla 1. Proyectos vinculados al proceso de investigación en el contexto del proyecto Simul@

\begin{tabular}{|c|c|c|}
\hline Proyecto & Datos Relevantes & Descripción \\
\hline \multirow{2}{*}{$\begin{array}{l}\text { Olimpiadas } \\
\text { Escolares }\end{array}$} & $\begin{array}{l}\text { Titulación: Magisterio especialidad } \\
\text { Educación Física } \\
\text { Asignatura: Fundamentos de } \\
\text { Deporte I }\end{array}$ & \multirow{2}{*}{$\begin{array}{l}\text { Organizar una Olimpiada Escolar de } \\
5 \text { días de duración, con un programa } \\
\text { deportivo y de actividades sociales } \\
\text { que recoja todas las especialidades } \\
\text { deportivas que oferta el centro. El } \\
\text { proyecto se tendrá que presentar y } \\
\text { defender ante un Comité Olímpico. }\end{array}$} \\
\hline & $\begin{array}{l}\text { Titulación: Grado de Educación } \\
\text { Asignatura: Procesos y Contextos } \\
\text { Educativos }\end{array}$ & \\
\hline $\begin{array}{l}\text { Organizar } \\
\text { Jornada } \\
\text { Escolar }\end{array}$ & $\begin{array}{l}\text { Titulación: Magisterio especialidad } \\
\text { en Educación Infantil } \\
\text { Asignatura: Organización del } \\
\text { Centro Escolar }\end{array}$ & $\begin{array}{l}\text { Organizar una jornada de una ZER } \\
\text { (instituciones escolares formadas } \\
\text { por escuelas rurales que comparten } \\
\text { el mismo proyecto educativo, las } \\
\text { mismas normas de organización, la } \\
\text { misma programación y los maestros } \\
\text { especialistas itinerantes), formada } \\
\text { por } 3 \text { escuelas, dos escuelas son } \\
\text { cíclicas (entre dos y cinco aulas) y } \\
\text { una unitaria. }\end{array}$ \\
\hline $\begin{array}{l}\text { Participación } \\
\text { en Ferias } \\
\text { Profesionales }\end{array}$ & $\begin{array}{l}\text { Titulación: Master Oficial de } \\
\text { Dirección Estratégica de Empresa } \\
\text { Asignatura: Dirección de } \\
\text { Marketing }\end{array}$ & $\begin{array}{l}\text { Participar en una feria de } 4 \text { días de } \\
\text { duración, para presentar y ofrecer } \\
\text { un nuevo producto y/o servicio. }\end{array}$ \\
\hline
\end{tabular}

Fuente: Simul@: Evaluación de un entorno tecnológico de simulación para el aprendizaje de competencias transversales en la universidad (Ref. EDU2008-01479) 
La secuencia didáctica seguida por los estudiantes está compuesta de cuatro fases:

1. Preliminar: La única que fue presencial, contempla un conjunto de acciones orientadas a introducir y familiarizar a los estudiantes en el uso de la plataforma tecnológica; considera actividades como la instalación del visor, personalización del avatar, explicación del proyecto, formación de grupos y el diagnóstico de los participantes.

2. Planificación: El grupo debe diseñar la maqueta de solución a implementar, para esto debía acumular puntos a través de la realización de actividades de tipo organizacional, presupuestos y calendarios.

3. Desarrollo/Construcción: El grupo debe gestionar recursos, obtener o desarrollar nuevos recursos y utilizarlos adecuadamente.

4. Evaluación: Consistió en la presentación del proyecto y el proceso de evaluación indirecta que contempla una autopercepción, la co-evaluación y la heteroevaluación.

Los participantes en las distintas fases desarrollaron algunas acciones en busca de alcanzar las metas planteadas en la actividad pedagógica (figura 2).

Figura 2. Trabajo colaborativo de los participantes en las distintas fases de la actividad formativa
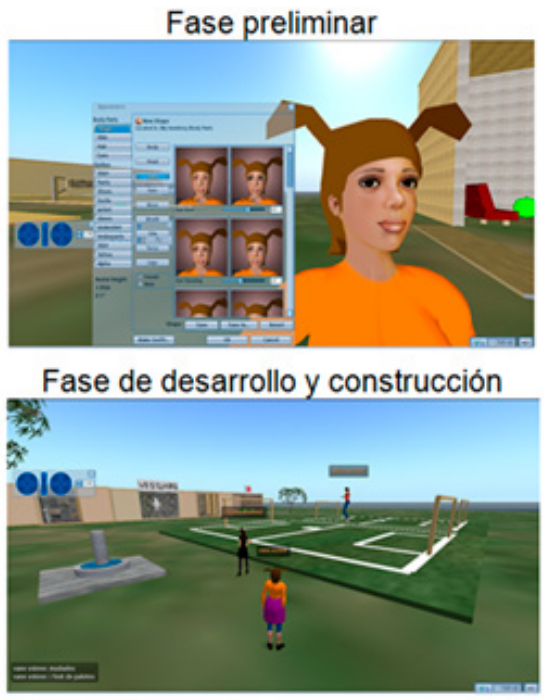
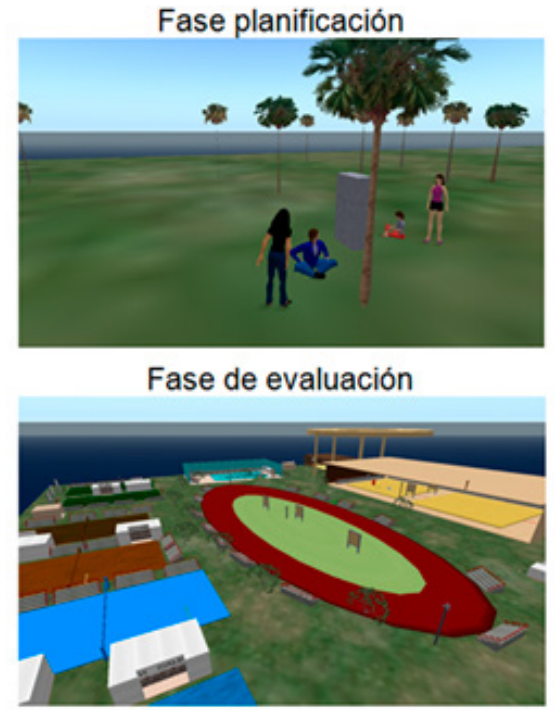


\section{Plataforma tecnológica}

En cuanto al entorno tecnológico, se utilizó una plataforma integrada remotamente al servidor Moodle y al OpenSim. La integración del Moodle con OpenSim es posible a través de la instalación y configuración del módulo de Sloodle en el servidor Moodle. Para acceder al entorno de simulación, se especificó la dirección IP del servidor OpenSim a través de su aplicación cliente (visor). Con la integración de la plataforma, los estudiantes trabajan en un entorno de simulación de OpemSim interactuando con sus objetos 3D, de manera que la interacción entre participantes y con los objetos sea registrada en el servidor Moodle.

\section{RESULTADOS}

El propósito de este apartado es observar la actuación de los estudiantes con patrones deaprendizajeStrong-Willedy Bridge seleccionados demaneraintencionada en busca de evidencias que nos permita describir su actuación en el contexto de la actividad formativa, la secuencia pedagógica y el entorno de simulación.

\section{Interacción colaborativa del perfil Strong-Willed}

En la tabla 2 y la figura 3 se presentan los resultados de las interacciones generadas por los estudiantes con perfil Strong-Willed en las diferentes fases y actividades que se desarrollaron en el contexto de cada proyecto.

Tabla 2. Interacción del perfil Strong-Willed con propósito colaborativo

\begin{tabular}{|c|c|c|c|c|c|c|c|}
\hline \multirow[b]{2}{*}{ Estudiante } & \multicolumn{3}{|c|}{ F. Planificación } & \multicolumn{2}{|c|}{ F. Desarrollo } & \multicolumn{2}{|c|}{ F. Evaluación } \\
\hline & $\begin{array}{l}\text { ORG_- } \\
\text { ACTIV }\end{array}$ & $\begin{array}{c}\text { ORG_- } \\
\text { PRESU }\end{array}$ & $\begin{array}{l}\text { CALC } \\
\text { ALTIV }\end{array}$ & $\begin{array}{c}\text { GES } \\
\text { RECUR }\end{array}$ & $\begin{array}{c}\text { OBT }_{-} \\
\text {RECUR }\end{array}$ & $\begin{array}{l}\text { AUT } \\
\text { PERCE }\end{array}$ & COA- \\
\hline IF & 30 & 16 & 5 & 22 & 5 & 20 & 2 \\
\hline $\mathbf{L P}$ & 49 & 7 & 5 & 20 & 11 & 34 & 12 \\
\hline SB & o & o & o & o & o & 1 & 1 \\
\hline MR & 63 & 16 & 13 & 48 & 22 & 25 & 14 \\
\hline TOTAL & 142 & 39 & 23 & 90 & 38 & 80 & 29 \\
\hline
\end{tabular}


Figura 3. Interacción con propósito colaborativo del perfil Strong-Willed por actividad

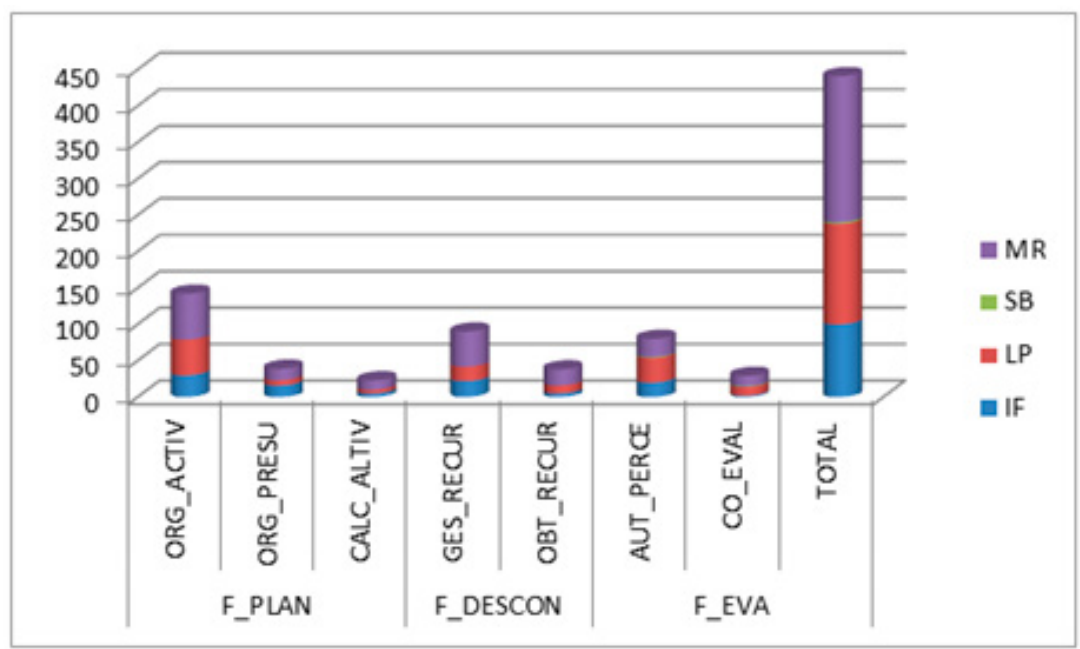

La mayor interacción con fines de colaboración del perfil Strong-Willed, en ese orden respectivamente, se presenta en las actividades Organizar Actividad, Gestionar Recursos y Auto Percepción. También, se observa que el perfil Strong-Willed genera interacción con propósito colaborativo en todas las fases de la secuencia pedagógica.

En la tabla 3, se presenta una muestra del tipo de interacciones que realizan los estudiantes con perfil Strong-Willed, a través de las cuales se caracteriza la actuación de los estudiantes con este perfil.

Tabla 3. Características del perfil Strong-Willed

\section{Organiza y estructura actividades:} Plantea y estructura actividades a desarrollarse en el grupo para alcanzar objetivos comunes.
MR: a veure hi ha tres ambits. [A.3.6_EV_G2_61]

MR: i cadascu den osaltres es responsable d'un ambit. [A.3.6_EV_G2_65]

IF: accions d difusio ja ho vma parlar per aki no?.[A.3.6_EV_G2_493]

IF: lo de com fem la publicitat i tot. [A.3.6_EV_G2_494]

LP: si, son 5 grups pos anem a fer una paret [A.3.5_CE_G4_128]

LP: i dsp vos dic latra activitat [A.3.5_CE_G4_129] 


\begin{tabular}{|c|c|}
\hline $\begin{array}{l}\text { Asume } \\
\text { responsabilidades: } \\
\text { En el desarrollo del } \\
\text { trabajo colaborativo } \\
\text { asume el desarrollo de } \\
\text { trabajo en el grupo de } \\
\text { manera autónoma. }\end{array}$ & $\begin{array}{l}\text { MR: sino la faig jo mateix. [A.3.6_EV_G2_53] } \\
\text { MR: o weno ja la faig jo i li paso. [A.3.6_EV_G2_170] } \\
\text { IF: home...trobo q hauria d'estar alla. [A.3.6_EV_G2_573] } \\
\text { IF: jo trobo q nhi hauria d'haver un per pista no?. [A.3.6_EV_G2_189] } \\
\text { LP: q faig acabo el } 6 \text { i ja esta? [A.3.5_CE_G4_419] } \\
\text { LP: pos io construio [A.3.5_CE_G4_434] }\end{array}$ \\
\hline $\begin{array}{l}\text { Distribuye } \\
\text { responsabilidades: } \\
\text { Distribuye el } \\
\text { desarrollo del trabajo } \\
\text { entre los demás } \\
\text { miembros del grupo. }\end{array}$ & $\begin{array}{l}\text { MR: pos leon has de crear una note [A.3.6_EV_G2_21] } \\
\text { MR: algu la vol fer? [A.3.6_EV_G2_52] } \\
\text { IF: pero aquesta l'hauria d'enviar leon q es el responsable d competicio } \\
\text { [A.3.6_EV_G2_528] } \\
\text { IF: i la 9 es cada una el seu. [A.3.6_EV_G2_224] } \\
\text { LP: i fan un taller de pintures rupestres : el mural [A.3.5_CE_G4_123] } \\
\text { LP: llucia q li va aixo [A.3.5_CE_G4_138] }\end{array}$ \\
\hline $\begin{array}{l}\text { Lidera el trabajo } \\
\text { del grupo: Es } \\
\text { el que marca la } \\
\text { pauta de cómo se } \\
\text { trabaja, propone y } \\
\text { dirige el trabajo del } \\
\text { grupo. En ocasiones } \\
\text { pregunta para recibir } \\
\text { retroalimentación } \\
\text { de lo que se está } \\
\text { haciendo. } \\
\end{array}$ & $\begin{array}{l}\text { MR: es a dir tots ens hem de repartir de tot. [A.3.6_EV_G2_6o] } \\
\text { MR: ara be, resposnbale no vol dir que nomes ho pogui fer ell. [A.3.6_ } \\
\text { EV_G2_67] } \\
\text { IF: si avere, esta clar q publici ateltes han d'entrar per separat no? [A.3.6_ } \\
\text { EV_G2_119] } \\
\text { IF: osigui que es parla per aqui no? [A.3.6_EV_G2_174] } \\
\text { LP: un x curs xo necessitem espais pq de cada curs se dividien en grups } \\
\text { [A.3.5_CE_G4_99] } \\
\text { LP: que fem? construim o qdem? [A.3.5_CE_G4_242] }\end{array}$ \\
\hline $\begin{array}{l}\text { Toma decisiones: } \\
\text { Es la persona que } \\
\text { decide sobre las } \\
\text { acciones que se deben } \\
\text { hacer en el grupo. }\end{array}$ & $\begin{array}{l}\text { MR: a veure esl esports que em posat. [A.3.6_EV_G2_509] } \\
\text { MR: Futbol, atletisme, natacio, volei handball, hoquei, básquet [A.3.6_ } \\
\text { EV_G2_510] } \\
\text { IF: i si aixo ja ho facada un quan vulgui i ara fem lo d grup? [A.3.6_EV_- } \\
\text { G2_312] } \\
\text { IF: espera q acabo amb la ntoa i continuem [A.3.6_EV_G2_433] } \\
\text { LP: avore esta molt be lo q estem fent [A.3.5_CE_G4_249] } \\
\text { LP: despues vos truquem i mos dieu que aclariu i ell i jo seguim fent } \\
\text { despres ok? [A.3.5_CE_G4_339] }\end{array}$ \\
\hline
\end{tabular}




\begin{tabular}{|c|c|}
\hline $\begin{array}{l}\text { Colabora con el } \\
\text { grupo de trabajo: } \\
\text { Apoya al grupo en } \\
\text { el desarrollo del } \\
\text { trabajo, apoyando } \\
\text { a la resolución de } \\
\text { problemas, aclarando } \\
\text { dudas, guiando y } \\
\text { encaminando el logro } \\
\text { de objetivos. }\end{array}$ & $\begin{array}{l}\text { MR: es al meeting point [A.3.6_EV_G2_303] } \\
\text { MR: ens ho preguntes?. [A.3.6_EV_G2_155] } \\
\text { IF: aixo ja no ho se, crec q nomes hem de dir que hi psoariem. [A.3.6_EV_ } \\
\text { G2_162] } \\
\text { IF: quins espots posariem al mati, quins a la tarde etc. [A.3.6_EV__ } \\
\text { G2_507] } \\
\text { LP: vale va, avore natros mon hem danar a les } 3 \text { [A.3.5_CE_G4_81] } \\
\text { LP: avore pensem que mos fa falta [A.3.5_CE_G4_94] }\end{array}$ \\
\hline $\begin{array}{l}\text { Controla la } \\
\text { participación } \\
\text { activa del grupo: } \\
\text { Hace seguimiento } \\
\text { de la participación } \\
\text { de los miembros del } \\
\text { grupo y demanda } \\
\text { su integración a } \\
\text { colaborar. }\end{array}$ & $\begin{array}{l}\text { MR: Ivet vols deixar el facE!. [A.3.6_EV_G2_128] } \\
\text { MR: el leon fa mes de mitja hora que esta al face i el pavo no ma contestat } \\
\text { pel xat i le rallat a donfu [A.3.6_EV_G2_609] } \\
\text { IF: pero s estic aki, q no he marxar en cap moment. [A.3.6_EV_G2_140] } \\
\text { IF: no, nose res de leon. [A.3.6_EV_G2_454] } \\
\text { LP: llucia trobo [A.3.5_CE_G4_180] } \\
\text { LP: al facebook están [A.3.5_CE_G4_559] }\end{array}$ \\
\hline $\begin{array}{l}\text { Temporaliza el } \\
\text { trabajo: Calendariza } \\
\text { y establece tiempos } \\
\text { para el desarrollo del } \\
\text { trabajo del grupo. }\end{array}$ & $\begin{array}{l}\text { MR: pero es que em sebmla que aixo ha d'estar acabar per avui. [A.3.6_ } \\
\text { EV_G2_359] } \\
\text { MR: si es pot segur el dijous que ve. [A.3.6_EV_G2_361] } \\
\text { IF: doncs demà continuem ales 11. [A.3.6_EV_G2_613] } \\
\text { IF: molt bé Marc, em esmbla perfecte la teva planificacio [A.3.6_EV_- } \\
\text { G2_130] } \\
\text { LP: son les 2imija casi venga [A.3.5_CE_G4_151] } \\
\text { LP: vos vaig dien les atres activitats o dspuesċ [A.3.5_CE_G4_152] }\end{array}$ \\
\hline $\begin{array}{l}\text { Proactivo: Identifica } \\
\text { las necesidades de } \\
\text { manera previsiva y } \\
\text { las gestiona en busca } \\
\text { de alternativas para } \\
\text { solventarlas. }\end{array}$ & $\begin{array}{l}\text { MR: calculem que tindrem uns ingressos de } 1500 \text { per patrocinaodr } \\
\text { [A.3.6_EV_G2_466] } \\
\text { MR: si ens queden totes les pistes fent una rotllana per dir-ho d'alguna } \\
\text { manera. [A.3.6_EV_G2_122] } \\
\text { IF: i uns 100o per fer publicitat. [A.3.6_EV_G2_484] } \\
\text { IF: pero el pessupost as de dir quins sos els ingresss i quis son els gstos. } \\
\text { [A.3.6_EV_G2_390] } \\
\text { LP: tenim 150 monedes m diu aquí [A.3.5_CE_G4_143] } \\
\text { LP: aixo pa infantil? pero necessitem un espai tipo una casa per a tenir } \\
\text { una paret no? [A.3.5_CE_G4_192] }\end{array}$ \\
\hline $\begin{array}{l}\text { Persistente y } \\
\text { constante en el } \\
\text { trabajo }\end{array}$ & $\begin{array}{l}\text { I Strong-Willed participa en todas las fases del } \\
\text { su persistencia y su constancia en el trabajo }\end{array}$ \\
\hline
\end{tabular}




\section{Interacción colaborativa del perfil Bridge}

En la tabla 4 y en la figura 4, se presentan los resultados de las interacciones generadas por los estudiantes con perfil Bridge en las diferentes fases y actividades que se desarrollaron en el contexto de cada proyecto.

Tabla 4. Interacción del perfil Bridge con propósito colaborativo

\begin{tabular}{|c|c|c|c|c|c|c|c|}
\hline \multirow[b]{2}{*}{ Estudiante } & \multicolumn{3}{|c|}{ F. Planificación } & \multicolumn{2}{|c|}{ F. Desarrollo } & \multicolumn{2}{|c|}{ F. Evaluación } \\
\hline & $\begin{array}{l}\mathrm{ORG}_{\overline{-}} \\
\text { ACTIV }\end{array}$ & $\begin{array}{l}\mathrm{ORG}_{-} \\
\text {PRESU }\end{array}$ & $\begin{array}{l}\text { CALC } \\
\text { ALTIV }\end{array}$ & $\begin{array}{l}\text { GES_- } \\
\text { RECUR }\end{array}$ & $\begin{array}{l}\mathrm{OBT}_{-} \\
\mathrm{RECUR}^{-}\end{array}$ & $\begin{array}{l}\mathrm{AUT}_{-} \\
\text {PERCE }\end{array}$ & CO_EVAL \\
\hline IF & 13 & 0 & o & 7 & 1 & 23 & 6 \\
\hline $\mathbf{L P}$ & 4 & o & o & 3 & o & 15 & 1 \\
\hline SB & 14 & 0 & o & 3 & o & 7 & 4 \\
\hline MR & 5 & 1 & o & 0 & o & 10 & 4 \\
\hline TOTAL & 36 & 1 & 0 & 13 & 1 & 55 & 15 \\
\hline
\end{tabular}

Figura 4. Interacción con propósito colaborativo de perfil Bridge

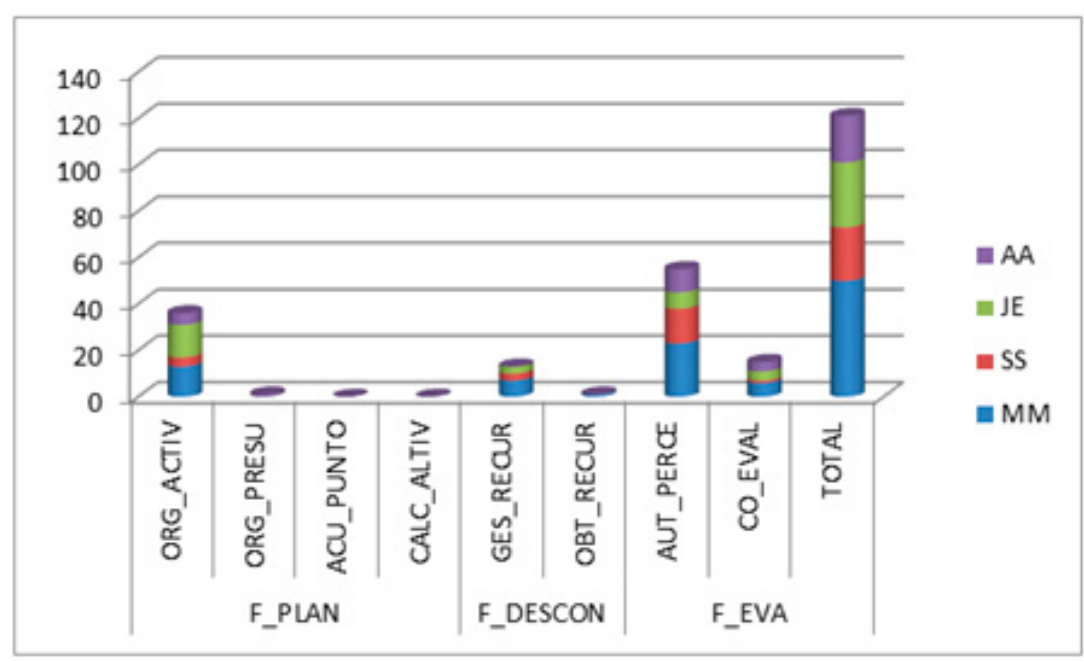

En los estudiantes con perfil Bridge, se observa que generan mayor interacción colaborativa en las actividades de Auto-percepción, Organiza Actividad y Gestiona 
Recursos. También, se observa que el perfil Bridge no genera interacción colaborativa en las actividades de Organiza Presupuesto y Calendariza Actividad.

En la tabla 5, se presenta una muestra del tipo de interacciones que realizan los estudiantes con perfil Bridge, a través de las cuales se caracteriza la actuación de los estudiantes con este perfil. Como se puede observar, las características vienen glosadas o avaladas por la evidencia en forma de citas textuales de las transcripciones de los chats analizadas.

Tabla 5. Características del perfil Bridge

\begin{tabular}{|c|c|}
\hline Características & Evidencias de la interacción colaborativa \\
\hline $\begin{array}{l}\text { Motiva al grupo: } \\
\text { Estimula al grupo cuando } \\
\text { se desarrolla algún trabajo } \\
\text { o se alcanza una meta. }\end{array}$ & $\begin{array}{l}\text { SS: es mas facil como en los sims [A.3.1_CE_G3_5] } \\
\text { SS: es entretenido [A.3.1_CE_G3_12] } \\
\text { AA: siii [A.3.3_EV_G3_229] } \\
\text { AA: perfecte [A.3.3_EV_G3_305] } \\
\text { JE: a vale [A3.2_EV_G1_9o] } \\
\text { JE: ja esta [A3.2_EV_G1_101] } \\
\text { MM: mol xulo[A3.3_FP_G2_214] } \\
\text { MM: me gusta [A3.3_FP_G2_39] }\end{array}$ \\
\hline $\begin{array}{l}\text { Se suma al trabajo } \\
\text { del grupo: Cuando } \\
\text { es requerido asume } \\
\text { responsabilidades y } \\
\text { colabora planteando } \\
\text { iniciativas en el desarrollo } \\
\text { del trabajo del grupo. }\end{array}$ & $\begin{array}{l}\text { SS: ja em canviare en tindre temps k stic molt enfeinada nena } \\
\text { XD [A.3.1_CE_G3_6] } \\
\text { SS: a la smbra d ls pinos...XD [A.3.1_CE_G3_18] } \\
\text { AA: i quins podem triar? [A.3.3_EV_G3_298] } \\
\text { AA: jo trobo k isostar es mes esportiva k les altres. [A.3.3_EV_ } \\
\text { G3_317] } \\
\text { JE: quan els acceptem dieu-ho [A3.2_EV_G1_31] } \\
\text { JE: Organitzar l'entrada i sortida de tots els assistents [A3.2_ } \\
\text { EV_G1_36] } \\
\text { MM: es lo qe necesitamos [A3.3_FP_G2_34] } \\
\text { MM: i esciure alguna koseta x les parets [A3.3_FP_G2_145] }\end{array}$ \\
\hline $\begin{array}{l}\text { Se apoya en su grupo: } \\
\text { Se apoya en el trabajo de } \\
\text { los compañeros del grupo } \\
\text { para aprender y resolver } \\
\text { cosas que desconoce cómo } \\
\text { hacerlas. }\end{array}$ & $\begin{array}{l}\text { SS: si ya te e visto kmo lo acias [A.3.1_CE_G3_21] } \\
\text { SS: dl nostre grup o en general?[A.3.1_CE_G3_37] } \\
\text { AA: ara on vaig? [A.3.3_EV_G3_252] } \\
\text { AA: quines heu fet? [A.3.3_EV_G3_257] } \\
\text { JE: llegir que? [A3.2_EV_G1_] } \\
\text { JE: k ja existeix no??[A3.2_EV_G1_] } \\
\text { MM: avere esplicam [A3.3_FP_G2_] } \\
\text { MM: ajjaja sus ideas [A3.3_FP_G2_] }\end{array}$ \\
\hline
\end{tabular}




\begin{tabular}{|c|c|}
\hline $\begin{array}{l}\text { Expresivo: Expresa sus } \\
\text { emociones y las transmite } \\
\text { al grupo de manera } \\
\text { espontánea. }\end{array}$ & $\begin{array}{l}\text { MM: qui es qui [A3.3_FP_G2_10] } \\
\text { MM: ye ye ye ye y [A3.3_FP_G2_19] } \\
\text { SS: bah ...estaran al meu favor xd [A.3.1_CE_G3_] } \\
\text { SS: ja els sobornare [A.3.1_CE_G3_] } \\
\text { AA: uf es molt llarg eh [A.3.3_EV_G3_] } \\
\text { AA: bona loren! [A.3.3_EV_G3_] } \\
\text { JE: pamplines [A3.2_EV_G1_] } \\
\text { JE: què us sembla? [A3.2_EV_G1_] }\end{array}$ \\
\hline $\begin{array}{l}\text { Comunicativo: } \\
\text { Comunica de manera } \\
\text { natural y simple los } \\
\text { sucesos de su entorno. }\end{array}$ & $\begin{array}{l}\text { SS: } x \text { eso dgo [A.3.1_CE_G3_15] } \\
\text { SS: tenen problemas [A.3.1_CE_G3_40] } \\
\text { AA: nomes veig les dues ultimes frases que has dit ara [A.3.3_ } \\
\text { EV_G3_277] } \\
\text { AA: vale he llegit una mica [A.3.3_EV_G3_290] } \\
\text { JE: me rebeu? [A3.2_EV_G1_13] } \\
\text { JE: algun més [A3.2_EV_G1_52] } \\
\text { MM: med recibes? [A3.3_FP_G2_17] } \\
\text { MM: no le dire anda a nadie [A3.3_FP_G2_46] }\end{array}$ \\
\hline $\begin{array}{l}\text { Intermitente en el } \\
\text { trabajo }\end{array}$ & $\begin{array}{l}\text { Se puede decir que es intermitente y poco constante en el } \\
\text { desarrollo del trabajo de grupo }\end{array}$ \\
\hline
\end{tabular}

\section{DISCUSIÓN Y CONCLUSIONES}

En esta investigación, se ha evidenciado que los entornos de simulación 3D con mediación pedagógica favorecen la comunicación, interacción y colaboración de los participantes del proceso formativo; estimulando la actividad de los participantes en el desarrollo de las actividades formativas. En el contexto de la actividad formativa y la secuencia pedagógica, se observa que se genera interacción con propósito de colaboración, donde los participantes aportan sus ideas y alternativas, realizan trabajo efectivo para la consecución de los objetivos del grupo.

También, se observa que el comportamiento y la actuación de los perfiles StrongWilled y Bridge son distintos. El perfil Strong-Willed se caracteriza por organizar, asumir y distribuir responsabilidades, tomar decisiones, colaborar, liderar el trabajo del grupo, ser persistente y constante. Sin embargo, el perfil Bridge se caracteriza por ser comunicativo y expresivo, se convierte en el motivador del grupo, se apoya en el conocimiento de los miembros del grupo para aprender y desarrollar sus actividades y carece de continuidad durante el desarrollo del trabajo grupal.

Una vez estudiada y analizada la actuación de los perfiles Strong-Willed y Bridge en las interacciones con propósito colaborativo, se observa que la aportación efectiva a través de las interacciones de los participantes no es la misma ni en calidad ni en cantidad; por tanto, existen diferencias entre cada participante, lo que permite 
reconocer su individualidad a la hora de aprender. El análisis de la comunicación de los perfiles específicos determinados en la muestra ha facilitado un resultado que añade valor significativo a la investigación en la medida que contribuye a la caracterización de estos dos perfiles (tabla 6).

Tabla 6. Aportación a las características de los perfiles Strong-Willed y Bridge

\begin{tabular}{|l|l|}
\hline \multicolumn{1}{|c|}{ Strong-Willed } & \multicolumn{1}{c|}{ Bridge } \\
\hline $\begin{array}{l}\text { Organiza y estructura actividades. } \\
\text { Asume Responsabilidades. } \\
\text { Distribuye Responsabilidades. } \\
\text { Lidera el trabajo en grupo. } \\
\text { Toma decisiones. }\end{array}$ & \\
Colabora con el grupo de trabajo. & Motiva al grupo. \\
Controla la participación activa del grupo. & Se suma al trabajo del grupo. \\
Temporaliza el trabajo. & Se apoya en el grupo. \\
Proactivo. & Comunicativo. \\
Persistente y constante en el trabajo. & Intermitente en el trabajo. \\
\hline
\end{tabular}

Además, se ha considerado conveniente utilizar esta estrategia para conformar grupos con estudiantes de diferentes perfiles para que integrados en el trabajo se apoyen y colaboren mutuamente durante el proceso de aprendizaje. Ligado a lo anterior, con el conocimiento de los diferentes patrones y perfiles de aprendizaje de los estudiantes, los profesores podrían impulsar sociedades para fomentar y combinar estrategias que permitan sacar el mejor provecho de las diferentes características de aprendizaje de los estudiantes en el proceso de enseñanza-aprendizaje.

Desde el quehacer docente, se encuentra una gran oportunidad para apoyar el desarrollo de actitudes y aptitudes, integrando al proceso formativo actividades que promuevan el aprendizaje activo en concordancia con los perfiles de aprendizaje, garantizando una mayor motivación a la hora de aprender.

\section{Agradecimientos}

Los autores desean agradecer a los revisores por sus valiosas aportaciones y a los docentes y estudiantes, quienes voluntariamente participaron en este estudio. Gracias a los investigadores del Applied Research Group in Education and Technology (ARGET) por toda su ayuda. 


\section{REFERENCIAS BIBLIOGRÁFICAS}

Cela-Ranilla, J. (2008). Personality, learning patterns and performance of first year students (Tesis doctoral). Hamburg: Universität Hamburg. Recuperado de http://ediss.sub.uni-hamburg.de/ volltexte/2010/4807/

Dawkins, B. U., Kottkamp, R. B., y Johnston, C. A. (2010). Intentional teaching: The let me learn $\AA$ classroom in action. Thousand Oaks, CA: Corwin Press. doi: http:// dx.doi.org/10.4135/9781452274669

Della Porta, D., y Keating, M. (2008). Approaches and methodologies in the social sciences: A pluralist perspective. Cambridge, UK: Cambridge University Press.

De Oliveira, J. M., Gallardo-Echenique, E., Bettencourt, T., y Gisbert, M. (2012). Meandros de la interacción: Desafíos del uso pedagógico de los entornos virtuales 3D [Monográfico Entornos Virtuales de Aprendizaje en Iberoamérica]. Revista Ibero-Americana de Educación, 6o, 153151.

Domínguez Rodríguez, E., y Alonso Díaz, L. (2012). La complementariedad de entornos virtuales síncronos y asíncronos de aprendizaje en los procesos formativos. In L. García Aretio (Ed.), Sociedad del conocimiento y educación (149154). Madrid: Universidad Nacional de Educación a Distancia (UNED).

Gallardo-Echenique, E. E. (2012). Hablemos de estudiantes digitales y no de nativos digitales. Universitas Tarraconensis, Revista de Ciències de l'Educació, 7-21.

Gallardo-Echenique, E. E., Marqués Molías, L., y Bullen, M. (2014). Usos académicos y sociales de las tecnologías digitales del estudiante universitario de primer año. Tendencias Pedagóqicas, 23, 191-204. Recuperado de http:/ www.tendenciaspedagogicas.com/ Articulos/2014 23 14.pdf
Guiza Ezkauriatza, M. (2011). Trabajo colaborativo en la web: Entorno virtual de autogestión para docentes (Tesis doctoral). Palma de Mallorca: Universitat de les Illes Balears.

Hernández, J., Pennesi, M., Sobrino, D., y Vázquez, A. (Coord.) (2013). Tendencias emergentes en Educación con TIC. Barcelona: Asociación Espiral, Educación y Tecnología.

Johnston, C. A. (1996). Unlocking the will to learn. Thousand Oaks, CA: Corwin Press.

Johnston, C. A., y Dainton, G. (1996). The learning combination inventory. Thousand Oaks, CA: Corwin Press.

Johnson, D. W., Johnson, R. T., y Johnson Holubec, E. (1994). The New Circles of Learning: Cooperation in the Classroom and School. Alexandria, VA: Association for Supervision and Curriculum Development (ASCD).

Johnson, D. W., y Johnson, R. T. (2004). Cooperation and the use of technology. In D. H. Jonassen (Ed.), Handbook of research on educational communications and technology. 2nd ed., (785-811). Mahwah, NJ: Lawrence Erlbaum Associates.

Lucero, M. M. (2003). Entre el trabajo colaborativo y el aprendizaje colaborativo. Revista Iberoamericana de Educación (RIE), 1-20. Recuperado de http://www. rieoei.org/tec edu18.htm

Maldonado Pérez, M. (2007). El trabajo colaborativo en el aula universitaria. Laurus, 13(23), 263-278. Recuperado de http://www.redalyc.org/ pdf/761/76102314.pdf

Martínez Sánchez, F., y Prendes Espinosa, M. P. (Eds.) (2004). Nuevas tecnologías y educación. Madrid: Pearson-Prentice Hall.

Maxwell, J. A. (2009). Designing a qualitative study. In L. Bickman y D. J. Rog (Eds.), The SAGE Handbook 
of Applied Social Research Methods. 2nd ed., (214-253). doi: http://dx.doi. org/10.4135/9781483348858

Medina Vidal, F., y Hernández Gómez, E. (2011). El aprendizaje colaborativo como herramienta para la innovación educativa en el aula de la Educación Secundaria Obligatoria. En Congreso Internacional de Innovación Docente (pp. 1943-1954). Cartagena: Universidad Politécnica de Cartagena.

Simul@ (2008). Evaluación de un entorno tecnológico de simulación para el aprendizaje de competencias transversales en la universidad (Ref. EDU2008-01479) Recuperado de http:/ late-dpedago.urv.cat/simula

Vaca-Barahona, B. E. (2012). Comunicación $y$ perfiles de aprendizaje en entornos de simulación $3 D$ (Master's Thesis). Tarragona: Universitat Rovira i Virgili.

Vega Cruz, P., Almaraz, F., Pinto Escribano, J., Ortega Mohedano, J., López Rodrigo, J., y Martín Hernández, S. (Eds.).
(2011). Aproximación pedagógica a las plataformas open source en la universidad española (Monográfico SCOPEO N ${ }^{\circ}$ 2). Salamanca: SCOPEO.

Villamizar Carrillo, L. (2006). Aplicación del sistema de aprendizaje Let Me Learn (R) en la formación técnica y didáctica en Tecnologías de Información y Comunicaciones (TIC's) de Profesores Universitarios: dos estudios de caso. Universitas Tarraconensis, Revista de Ciències de l'Educació, 233-246. Recuperado de http://pedagogia.fcep.urv. cat/revistaut/revistes/junyo6/article11. pdf

Villamizar Carrillo, L., González Soto, Á.-P., y Gan Acosta, A. (2008). Uso e importancia de las Tecnologías de Información y Comunicaciones (TICS) en la docencia universitaria: Un estudio de caso utilizando Let Me Learn. Revista Colombiana de Tecnologías de Avanzada, 1(11), 92-97.

\section{PERFIL ACADÉMICO Y PROFESIONAL DE LOS AUTORES}

Byron Vaca-Barahona. Doctor en Tecnologías Educativas: e-learning y gestión del conocimiento en la Universidad Rovira i Virgili (España). Miembro del grupo de investigación "Applied Research Group in Education and Technology (ARGET)". Principales áreas de investigación: entornos de simulación en $3 \mathrm{D}$, comunidades virtuales de aprendizaje, trabajo colaborativo, perfiles de aprendizaje, ingeniería de software, seguridades informáticas, e-learning, desarrollo de aplicaciones informáticas, software libre.

E-mail: bvacab@espoch.edu.ed

\section{DIRECCIÓN DEL AUTOR}

Escuela de Ingeniería de Sistemas

Escuela Superior Politécnica de Chimborazo

Panamericana Sur Km. 1 1/2

Riobamba (Ecuador) 
Jose Cela-Ranilla. Doctor en Educación en la Universidad de Hamburgo (Alemania). Miembro del grupo de investigación "Applied Research Group in Education and Technology (ARGET)". Principales áreas de investigación: entornos de simulación en $3 \mathrm{D}$, comunidades virtuales de aprendizaje, trabajo colaborativo, trabajo en equipo, perfiles de aprendizaje.

E-mail: iosemaria.cela@urv.cat

\section{DIRECCIÓN DEL AUTOR}

Departamento de Pedagogía

Universitat Rovira i Virgili

Campus Sescelades. Carretera de Valls s/n.

Tarragona (España)

Eliana Esther Gallardo-Echenique. Doctora en Tecnologías Educativas: e-learning y gestión del conocimiento en la Universidad Rovira i Virgili (España). Miembro del grupo de investigación "Applied Research Group in Education and Technology (ARGET)”. Principales áreas de investigación: competencia digital, estudiante digital, e-learning, trabajo colaborativo, entornos de simulación en $3 \mathrm{D}$, juegos educativos, tecnologías digitales, métodos cualitativos, método mixto.

E-mail: egallardo@continental.edu.pe

\section{DIRECCIÓN DE LA AUTORA}

Oficina de Virtualización de Contenidos

Universidad Continental

Av. San Carlos 1980

Huancayo (Perú)

Fecha de recepción del artículo: 19/10/2015

Fecha de aceptación del artículo: 21/01/2016

\section{Como citar este artículo:}

Vaca-Barahona, B. E., Cela-Ranilla, J., y Gallardo-Echenique, E. E. (2016). Perfiles de aprendizaje y trabajo colaborativo en entornos de simulación en $3 \mathrm{D}$. RIED. Revista Iberoamericana de Educación a Distancia, 19(2), pp. 193-215. doi: http://dx.doi.org/10.5944/ried.19.2.15438 